\title{
Prostate Cancer Delineation in MRI Images Based on Deep Learning: Quantitative Comparison and Promising Perspective
}

\author{
Eddardaa BEN LOUSSAIEF ${ }^{\mathrm{a}, 1}$, Mohamed ABDEL-NASSER ${ }^{\mathrm{a}}$ and Domènec PUIG $^{\mathrm{a}}$ \\ ${ }^{a}$ Department of Computer Engineering and Mathematics, Universitat Rovira $i$ Virgili, \\ 43007 Tarragona, Spain
}

\begin{abstract}
Prostate cancer is the most common malignant male tumor. Magnetic Resonance Imaging (MRI) plays a crucial role in the detection, diagnosis, and treatment of prostate cancer diseases. Computer-aided diagnosis systems can help doctors to analyze MRI images and detect prostate cancer earlier. One of the key stages of prostate cancer CAD systems is the automatic delineation of the prostate. Deep learning has recently demonstrated promising segmentation results with medical images. The purpose of this paper is to compare the state-of-the-art of deep learning-based approaches for prostate delineation in MRI images and discussing their limitations and strengths. Besides, we introduce a promising perspective for prostate tumor classification in MRI images. This perspective includes the use of the best segmentation model to detect the prostate tumors in MRI images. Then, we will employ the segmented images to extract the radiomics features that will be used to discriminate benign or malignant prostate tumors.
\end{abstract}

Keywords. Prostate cancer, MRI images, image segmentation, deep learning.

\section{Introduction}

Prostate cancer is the most common type of cancer among men worldwide. There were 1.3 million new cases only in 2018. The highest rates of prostate cancer in 2018 were mainly in the European countries. Age-adjusted incidence rates of prostate cancer have increased dramatically and this is large because of the increased availability of screening for prostate-specific antigen (PSA) which were conducted for men without symptoms of the disease. This screening leads to the detection of many prostate tumors that are small or would otherwise remain unrecognized, and which may or may not develop further into higher stage disease. Accurate delineation of prostate cancer using medical scanners plays a crucial role in prostate diseases diagnosis and treatment.

\footnotetext{
${ }^{1}$ Department of Computer Engineering and Mathematics, Universitat Rovira i Virgili, 43007 Tarragona, Spain; E-mail: eddardaa.eniso@gmail.com.
} 
Previous research aimed at developing Computer-Aided Diagnosis (CAD) systems for Prostate cancer detection, classification, and prognostics using different medical imaging modalities, such as Computed Tomography (CT), Magnetic Resonance Imaging (MRI), and histopathological images. Early attempts prove that convolutional neural networks (CNNs) have achieved remarkable progress in many fields, particularly in computer vision and image understanding. Many researchers have also used CNNs to segment the prostate from MRI images. For instance, Milletari et al. [1] proposed a V-Net fully convolutional network $(\mathrm{FCN})$ with the Dice loss for accurate prostate segmentation. Yu et al. [2] introduced a novel network that incorporates boundary estimation, feature extraction, and shapes prior to prostate detection.

Regarding the pitfalls of deep learning architectures or the data acquisition strategy of medical imaging. One could find that the literature is still discussing the potentials of deep learning-based approaches for medical image segmentation. Regardless of the progress achieved, automated prostate segmentation remains challenging and the essential part of the research has not been fully addressed. As it is noted that the outcomes do not fully fit the clinical needs in terms of the accuracy and the precision rate to detect cancer in an earlier stage.

This paper aims at proposing a comparison between state-of-the-art deep learning segmentation models for prostate cancer delineation in MRI images. We highlight the limitations and strengths of each segmentation model. We train the segmentation model on three public datasets: Promise12, ISBI Challenge2013, and ProstateX. DSC coefficient and Hausdorff Distance evaluation metrics are used to assess the performance of the prostate cancer delineation models. This paper presents the first stage of our prostate cancer CAD system. Our perspective consists of the use of the best segmentation model to detect the prostate tumors in MRI images. Then, we will employ the segmented images to extract the radiomics features that will be used to discriminate benign or malignant prostate tumors.

The remainder of the paper is structured as follows. Section 2 provides a review of literature. Section 3 describes the proposed methodology in detail and highlights our perspective. In section 4, provides various experiments on prostate tumor segmentation and detection. Finally, we present our conclusion and introduce future remarks in the last Section 5 .

\section{Related Work}

Previous research has established promising results for automated prostate tumor segmentation. The developed methods can be mainly categorized into three classes: deformable methods, multi-atlas-based methods, and learning-based methods [1][2]. Firstly, deformable methods aim to accurately delineate the prostate, Toth et al. [4] introduced improved active appearance models (AAMs). Klein et al. [3] used atlas matching for prostate segmentation. The principal idea is to regroup the segmented images with target images and then fuse the aligned segmentation to reach the final results. Several deep-learning-based methods currently exist for automated prostate tumor delineation due to its automatic representation learning. Below, we present the $2 \mathrm{D}$ and $3 \mathrm{D}$ prostate segmentation methods. 


\subsection{D prostate segmentation methods}

Studies over the past decade have proved that the use of Convolutional Neural Networks (CNNs) have achieved their goals in many domains, particularly in computer vision[5][6] and medical imaging analysis. Automated segmentation is one of the pillars of medical image analysis, many researchers have explored the power of $\mathrm{CNN}$ in prostate cancer segmentation [7] [9]. The U-Net [8] architecture is one of the most popular networks for medical imaging segmentation. It uses a CNN as a downward sampling path with an up-sampling operation to boost the resolution of the output feature maps. Furthermore, It uses skip connections to transfer more information to the feature maps that are placed within the up-sampling operations [8]. This could be noted in the work of Clark et al [10], who is considered to be among the first researchers who used the U-net to segment the whole prostate. The U-net has been adapted to segment the whole prostate gland and transitional zone in Diffusion-Weighted MRI (DWI). They were able to achieve promising results on an in-house dataset. However, when they tested their approach on T2weighted (T2W) MRI data from the Promise 12 challenge [11] they were not able to achieve good accuracy.

Zhu et al. [12] proposed a deeply supervised U-Net architecture where they adjusted the number of convolution layers. The role of the deep supervision strategy is to supervise the hidden layers within the network and propagate them to the lower levels of the network. Also, they introduced residual blocks to reduce the number of hyper-parameters compared to the original U-Net. Tian et al. [13] implemented a PSNET that uses fully connected networks [14]. They tested their model on an in-house dataset consisting of 42 T2W MRI volumes and on two open-source prostate datasets: the ISBI2013 challenge [15] and the PROMISE12 challenge [11] datasets. Karimi et al. [16] used a smaller FCN consisting of three layers and validated it using a cross-validation scheme on a smaller dataset containing 49 T2W axial MRI images and 26 MRI images from the PROMISE12 challenge [11]. With this smaller network, Karimi et al. achieved a high DSC on their validation dataset.

In [17], Yoo et al. presented an automated CNN-based pipeline for detecting prostate cancer for an axial DWI (Diffusion-weighted imaging) image and each patient separately. Their proposed pipeline includes three stages. The first stage consists of classifying each DWI slice using five individually trained CNNs models. The second stage extracts the first-order statistical features (e.g., mean, standard deviation, median) from the CNNs outputs. Then, the relevant features are selected via a decision tree-based feature selector. In the last stage, they used a Random Forest classifier to classify patients. They used first-order statistical features to discriminate patients into sets with and without prostate cancer. Yu et al. [2] added a residual connection to the U-net network, which improves the prostate segmentation by using a sum operation instead of the concatenation operation features into the up-sampling layer. It turns the model into a hybrid model called ResNetU-Net. To boost the accuracy of prostate delineation, Zhu et al. [18] used a cascaded U-Net, where the role of the first network is to segment the whole prostate gland, and the obtained segmented gland was fed into the last network to detect the peripheral zone.

Liu et al. [20] used an FCN with ResNet50 as the backbone of their network to detect the prostate zones with the mechanism of feature pyramid attention to capture 
relevant semantic information at multiple scales. The results obtained by the features pyramid attention procedure are combined to generate a high-resolution feature representation, which improves the segmentation results over the original U-Net [8]. To ameliorate the prostate's delineation, Nie and Shen Nie [21] introduced a semantic guided strategy to learn discriminative features. They used a soft contour constraint mechanism to model the blurry boundary and trained their network using the $5 \mathrm{k}$-fold cross-validation on 50 prostate cancer patients with ground truths annotated manually of the rectum, prostate, and bladder. Their results were promising and outperformed state-of-the-art techniques. Zhu et al.[22] proposed a novel boundary-weighted segmentation loss that boosts the accuracy of the boundary segmentation. They also used a boundary-weighted transfer learning approach (domain adaptation) to surmount the restriction of small training datasets. Thanks to this strategy and several datasets as the source and target domain, they achieved state-of-the-art results. In [25], the authors used a channel-wise feature recalibration and integrated squeeze and excitation blocks into their network to enhance the segmentation results [25]. Their work performed well when they trained the model on all of the introduced datasets, but their network was less robust using some datasets.

To alleviate the issue of limitation of the training datasets and obtain a large amount of training data for creating a robust segmentation model, Liu et al. [27] implemented a multi-site network by aggregating prostate MRIs from multiple sites. Their network was able to learn universal representation across heterogeneous MRI scanners and images. Furthermore, they introduced Domain-Specific Batch normalization layers to enable the network to estimate the statistics and perform normalization for each site it had been trained on, separately. David Gillespie et al. [30] proposed a review for deep learningbased methods for prostate segmentation in MRI images and discussed their limitations and strengths. To improve the prostate segmentation, they introduced an optimized 2D U-net that uses the Ranger optimizer [28] and Mish Activation [29].

\section{2. $3 D$ prostate segmentation methods}

To accurately segment the prostate in MRI images, Milletari et al. [1] introduced a volumetric CNN based on V-shape fully convolutional networks (FCN). They adapted the U-Net architecture to segment prostates from 3D MRI volumes. They used Promise 12 datasets to train their network. They showed that their network performed significantly better when they applied a data augmentation procedure as preprocessing step on the datasets. To improve the segmentation accuracy of 3D volumes, Wang et al. [19] introduced a novel deeply supervised model into a 3D U-Net with group dilated convolutions to automatically segment the prostate gland in MRI scans. The main reason to use the deep supervision mechanism is to avoid exploding or vanishing gradients during the training stage of deep models, which forces the hidden layers filters to support highly discriminate features. A group of dilated convolutions was used to extract more global contextual information, as they extend the receptive field of the network. They also used a combined loss function including cosine and cross-entropy that evaluates the similarity and dissimilarity between segmented and manual contours. Zavala Romero et al. [26] developed a 3D multi-stream U-Net and proposed data prepossessing to normalize the data given from each MRI vendor. They implemented six models (for each vendor and combined vendor datasets). The results demonstrated that the combined vendor models 
performed better in the case of peripheral zone detection. However, for detecting the whole prostate from MRI images the individual data models performed better.

To extract a piece of relevant information from the Z-axis, a 3D U-Net network was proposed by Isensee et al [23]. But, 3D-Unet leads to the use of small training data because it needs a performant memory for computation. To tackle this issue, Isensee et al [23] proposed a novel method that compresses domain knowledge and chooses the best nnU-Net (2D or 3D) model for a given dataset. Using 5 fold cross-validation scheme, they trained a 2D and a 3D variant nnU-net on the Promise 12 challenge [11] and the Medical Decathlon dataset [24]. One common restriction of the mentioned work is that Isensee et al used a single site Prostate MRI scans to train their models, and get promising results on a private dataset. But it may not perform well on datasets from various sites or MRI vendors. This is a crucial challenge for prostate segmentation, as there are multiple MRI scanner vendors and protocols.

\section{Methodology}

Figure 1 represents an overview of the proposed methodology for this paper and our future work. The objective of this paper is to introduce a quantitative comparison between deep learning-based methods for prostate segmentation. Our main attempt is to train the deep learning models to segment prostate cancer from MRI scans. In this stage, we selected a set of public datasets to train and test the developed models such as Promise12 [11], ISBI2013 [15], and ProstateX [31]. Our strategy depends on the use of 2D and 3D segmentation models. As several medical image analysis studies used the U-Net architecture, we train the U-Net [8] and 2D-Unet [30] models for the 2D segmentation. For 3D segmentation, we train 3DFCN [1], 3D-Unet [23], and MS-Net [27].

All the architectures chosen above are employed in the literature. However, we applied data augmentation on the MRI datasets. We employed a resizing procedure to set the same slices number for each patient. Also, we set the same dimensions for all slices. Then, we split the dataset into training and testing data. To evaluate the performance of the segmentation models, we compute the Dice coefficient and Hausdorff distance. We display the results in the format of mean+std.

It should be noted that we perform the testing of the segmentation model to delineate prostate tumors in MRI images using the ProstateX [31] dataset. This prepares for our final goal that consists of the use of the obtained segmented images with their original MRI imaging to extract a set of radiomics features. Further, we will develop a classification model that adopts the extracted radiomics to discriminate between benign or malignant tumors. 


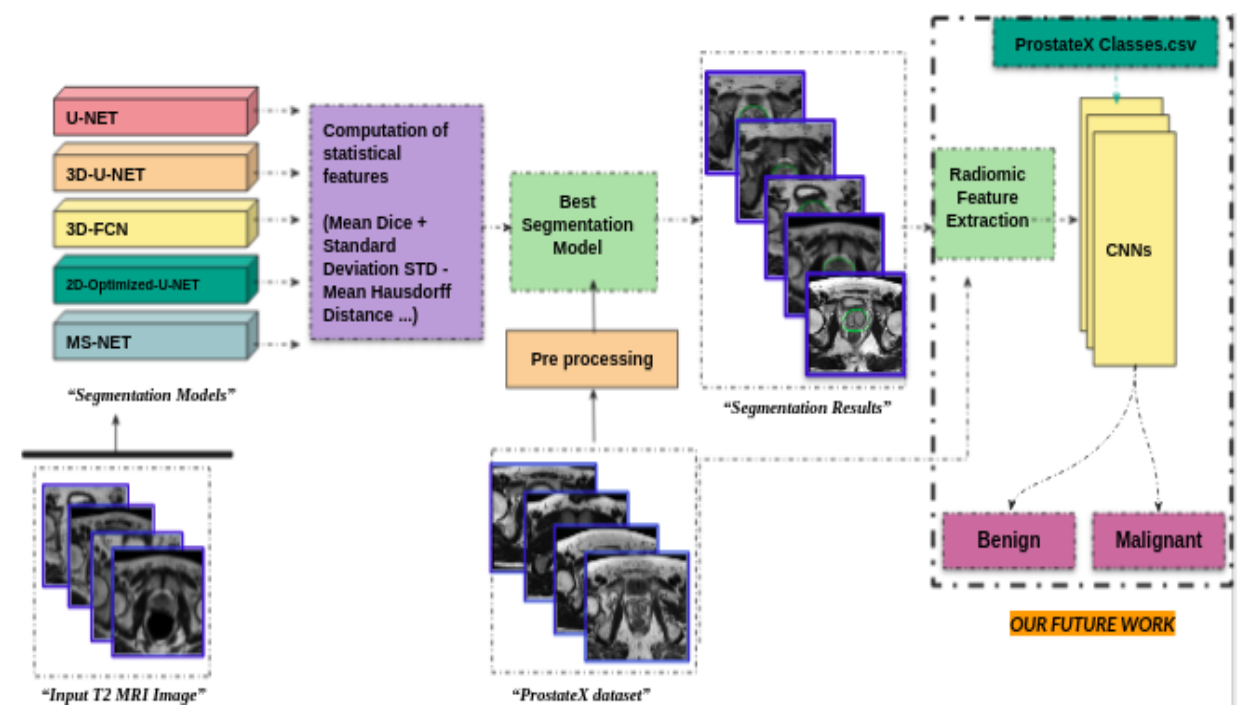

Figure 1. The schematic illustration of the proposed methodology. The first stage consists of training several deep learning-based methods for prostate delineation. Then testing the best segmentation model using the ProstateX dataset. The second stage stands for our future work that uses the segmentation results to extract a set of quantitative features in order to employ them in the classification of prostate tumors into benign or malignant.

\section{Experimentation and results}

\subsection{Datasets}

- PROMISE12 challenge [11] is a benchmark for evaluating segmentation algorithms of the MRI prostate. It contains a total of 50 patients transversal T2weighted MR images of the prostate and the corresponding true mask segmentation acquired in different hospitals that and variations in voxel size, dynamic range, position, field of view, and anatomic appearance, use a variety of vendors and acquisition protocols.e

- ISBI2013 challenge [15] consists of 60 patient scans saved in Dicom files (1.5 MRI and T3 MRI). The scans were acquired from Radboud University Nijmegen Medical Centre [RUNMC], Netherland. The ground truth for segmentation has been created by Drs. Nicolas Bloch (Boston University School of Medicine), Mirabela Rusu (Case Western University), Drs. Henkjan Huisman, Geert Litjens, Futterer at RUNMC.

- ProstateX Challenge [31] focused on quantitative image analysis methods for the diagnostic classification of clinically significant prostate cancer. The largest mpMRI dataset contains 346 participants and 349 studies. It consists of a total of 204 and 140 mpMRIs for training and testing respectively. The mpMRIs dataset includes the following sequences: T2-weighted (T2), diffusion-weighted (DW), apparent diffusion coefficient (ADC) map, and $\mathrm{K}$ trans (computed from dynamic contrast-enhanced -DCE- T1-weighted series). 


\subsection{Segmentation Results}

To evaluate the performance of several the segmentation models, we trained them on Promise12 [11] and ISBI2013 [15] datasets. We present in Table 1 and Table 2 the segmentation results in terms of the Dice coefficient and Hausdorff Distance.

Table 1. Comparing the performance of the segmentation models using ISBI2013 challenge dataset.

\begin{tabular}{c|c|c}
\hline \hline Model & Dice \pm STD & Hausdorff Distance \pm STD (mm) \\
\hline MS-Net [27] & $0.899 \pm 1.960$ & $9.511 \pm 4.011$ \\
\hline 2D-Unet [30] & $0.901 \pm 0.015$ & $6.030 \pm 3.082$ \\
\hline 3D-Unet [27] & $0.722 \pm 0.020$ & $17.761 \pm 2.924$ \\
\hline \hline
\end{tabular}

Figure 2 and Figure 3 present the qualitative results of the segmentation models with the ISBI2013 and Promise12 datasets, respectively.

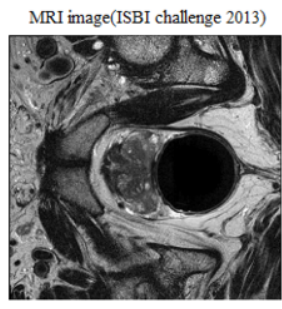

(a)

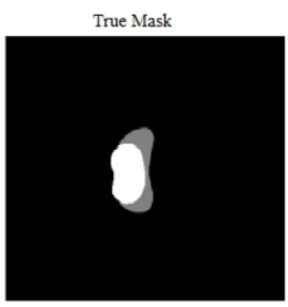

(b)

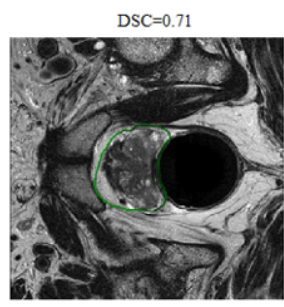

(c)

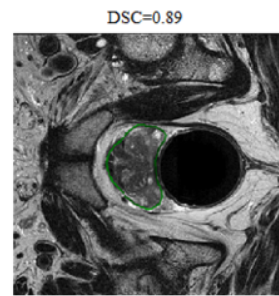

(d)

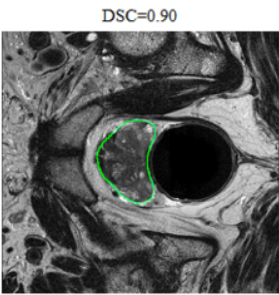

(f)

2013 chllenge.png 2013 chllenge.png

Figure 2. Segmentation results on ISBI 2013 Dataset. In the top row, from left to right, the raw image, ground truth, the segmentation results of 3D-Unet, respectively. In the button row, the segmentation results of MS-Net in the left, and the segmentation results of optimized 2D-Unet in the right.

Table 2. Comparing the performance of the segmentation models using PROMISE12 challenge dataset.

\begin{tabular}{c|c|c}
\hline \hline Model & Dice \pm STD & Hausdorff Distance \pm STD (mm) \\
\hline U-Net [8] & $0.880 \pm 0.041$ & $17.690 \pm 2.087$ \\
\hline 3D-FCN [1] & $0.790 \pm 0.050$ & $12.910 \pm 4.005$ \\
\hline 2D-Unet [30] & $0.899 \pm 0.021$ & $7.661 \pm 3.924$ \\
\hline \hline
\end{tabular}


MRI image (Promise12)

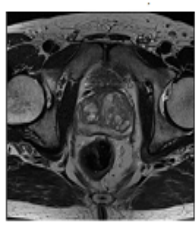

True Mask

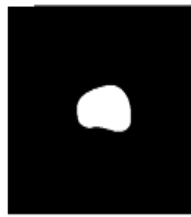

$\mathrm{DSC}=0.79$

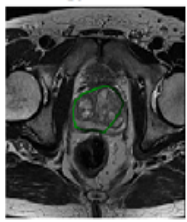

$\mathrm{DSC}=0.87$

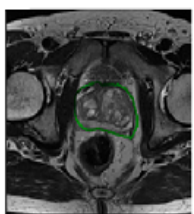

$\mathrm{DSC}=0.90$

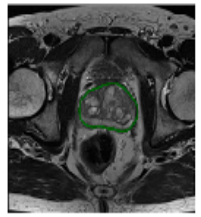

Figure 3. Segmentation results on the Promise 12 dataset. From left to right, the raw image, ground truth, the segmentation results of 3D-FCN, and the segmentation results of U-Net, respectively.

The results presented in the Table 1 and Table 2 demonstrate that the 2D-optimised Unet [30] provides the best dice coefficient with the ISBI and Promise12 datasets. The 2D-Unet model achieves a Hausdorff Distance (HD) of $6.03 \mathrm{~mm}$. These results demonstrate that 2D-Unet model can give accurate segmentation results on ProsateX Dataset. We show a qualitative segmentation example in Figure 4.

MRI Image (ProstateX Challenge)

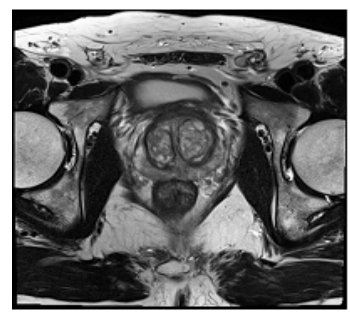

True Mask

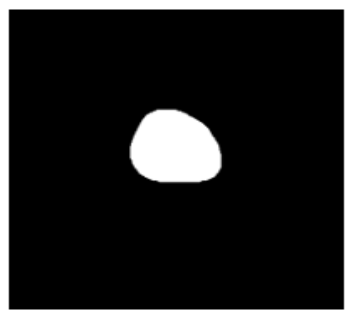

$\mathrm{DSC}=0.91$

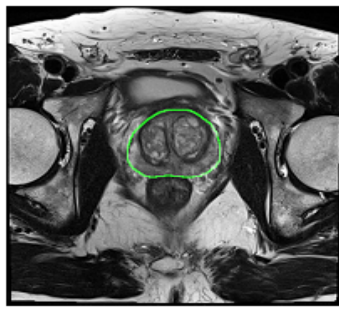

Figure 4. Segmentation results on the ProstateX dataset using 2D-Unet. From left to right, the raw image, ground truth and the segmentation result using $2 \mathrm{D}-\mathrm{UNet}$, respectively.

Table 3 presents the segmentation results when testing the segmentation models mentioned above on the ProstateX dataset.

Table 3. Quantitative segmentation result on prostatex dataset.

\begin{tabular}{c|c|c}
\hline \hline Model & Dice \pm STD & Hausdorff Distance \pm STD (mm) \\
\hline U-Net [30] & $0.791 \pm 0.151$ & $17.020 \pm 2.884$ \\
\hline 3D-UNet [30] & $0.701 \pm 0.078$ & $18.001 \pm 3.108$ \\
\hline 3D-FCN [1] & $0.721 \pm 0.047$ & $13.411 \pm 5.264$ \\
\hline 2U-Net [30] & $0.898 \pm 0.051$ & $7.690 \pm 2.987$ \\
\hline \hline
\end{tabular}

\section{Conclusion and Future Work}

This paper presented a comparative study for state-of-the-art deep learning-based segmentation models (U-net, 2D-Unet, 3D-Unet, Ms-Net and 3DFCN) for prostate delineation in MRI images. Different metrics were used to compare the prostate delineation models like the DSC coefficient and Hausdorff Distance. In future work, we will use 
the segmentation results to extract a set of radiomics to be inputted into a classifier to discriminate between prostate cancer classes (e.g., benign or malignant).

\section{Acknowledgement}

The Spanish Government partly supported this research through Project PID2019105789RB-I00.

\section{References}

[1] Milletari F, Navab N, Ahmadi SA. V-net: Fully convolutional neural networks for volumetric medical image segmentation. In2016 fourth international conference on 3D vision (3DV) 2016 Oct 25 (pp. 565571). IEEE.

[2] Yu L, Yang X, Chen H, Qin J, Heng PA. Volumetric ConvNets with mixed residual connections for automated prostate segmentation from 3D MR images. InProceedings of the AAAI Conference on Artificial Intelligence 2017 Feb 10 (Vol. 31, No. 1).

[3] Klein S, van der Heide UA, Lips IM, van Vulpen M, Staring M, Pluim JP. Automatic segmentation of the prostate in 3D MR images by atlas matching using localized mutual information. Med Phys. 2008 Apr;35(4):1407-17. doi: 10.1118/1.2842076. PMID: 18491536.

[4] Toth R, Madabhushi A. Multifeature landmark-free active appearance models: application to prostate MRI segmentation. IEEE Trans Med Imaging. 2012 Aug;31(8):1638-50. doi: 10.1109/TMI.2012.2201498. Epub 2012 May 30. PMID: 22665505.

[5] Simonyan K, Zisserman A. Very deep convolutional networks for large-scale image recognition. arXiv preprint arXiv:1409.1556. 2014 Sep 4.

[6] Szegedy C, Liu W, Jia Y, Sermanet P, Reed S, Anguelov D, Erhan D, Vanhoucke V, Rabinovich A. Going deeper with convolutions. InProceedings of the IEEE conference on computer vision and pattern recognition 2015 (pp. 1-9).

[7] Meyer A, Mehrtash A, Rak M, Schindele D, Schostak M, Tempany C, Kapur T, Abolmaesumi P, Fedorov A, Hansen C. Automatic high resolution segmentation of the prostate from multi-planar MRI. In2018 IEEE 15th International Symposium on Biomedical Imaging (ISBI 2018) 2018 Apr 4 (pp. 177-181). IEEE.

[8] Ronneberger O, Fischer P, Brox T. U-net: Convolutional networks for biomedical image segmentation. InInternational Conference on Medical image computing and computer-assisted intervention 2015 Oct 5 (pp. 234-241). Springer, Cham.

[9] Cheng R, Roth HR, Lu L, Wang S, Turkbey B, Gandler W, McCreedy ES, Agarwal HK, Choyke P, Summers RM, McAuliffe MJ. Active appearance model and deep learning for more accurate prostate segmentation on MRI. InMedical imaging 2016: Image processing 2016 Mar 21 (Vol. 9784, p. 97842I). International Society for Optics and Photonics.

[10] Clark T, Wong A, Haider MA, Khalvati F. Fully deep convolutional neural networks for segmentation of the prostate gland in diffusion-weighted MR images. InInternational Conference Image Analysis and Recognition 2017 Jul 5 (pp. 97-104). Springer, Cham.

[11] Litjens G, Toth R, van de Ven W, Hoeks C, Kerkstra S, van Ginneken B, Vincent G, Guillard G, Birbeck N, Zhang J, Strand R. Evaluation of prostate segmeZhu Q, Du B, Turkbey B, Choyke PL, Yan P. Deeplysupervised CNN for prostate segmentation. In2017 international joint conference on neural networks (IJCNN) 2017 May 14 (pp. 178-184). IEEE.ntation algorithms for MRI: the PROMISE12 challenge. Medical image analysis. 2014 Feb 1;18(2):359-73.

[12] Zhu Q, Du B, Turkbey B, Choyke PL, Yan P. Deeply-supervised CNN for prostate segmentation. In2017 international joint conference on neural networks (IJCNN) 2017 May 14 (pp. 178-184). IEEE.

[13] Tian Z, Liu L, Zhang Z, Fei B. PSNet: prostate segmentation on MRI based on a convolutional neural network. Journal of Medical Imaging. 2018 Jan;5(2):021208.

[14] Long J, Shelhamer E, Darrell T. Fully convolutional networks for semantic segmentation. InProceedings of the IEEE conference on computer vision and pattern recognition 2015 (pp. 3431-3440). 
[15] Nicholas Bloch AM. NCI-ISBI 2013 Challenge: Automated Segmentation of Prostate Structures [Internet]. The Cancer Imaging Archive; 2015. Available from: https://wiki.cancerimagingarchive.net/x/B4NEAQ

[16] Karimi D, Samei G, Kesch C, Nir G, Salcudean SE. Prostate segmentation in MRI using a convolutional neural network architecture and training strategy based on statistical shape models. International journal of computer assisted radiology and surgery. 2018 Aug;13(8):1211-9.

[17] Yoo S, Gujrathi I, Haider MA, Khalvati F. Prostate cancer detection using deep convolutional neural networks. Scientific reports. 2019 Dec 20;9(1):1-0.

[18] Zhu Y, Wei R, Gao G, Ding L, Zhang X, Wang X, Zhang J. Fully automatic segmentation on prostate MR images based on cascaded fully convolution network. Journal of Magnetic Resonance Imaging. 2019 Apr;49(4):1149-56.

[19] Wang B, Lei Y, Tian S, Wang T, Liu Y, Patel P, Jani AB, Mao H, Curran WJ, Liu T, Yang X. Deeply supervised 3D fully convolutional networks with group dilated convolution for automatic MRI prostate segmentation. Medical physics. 2019 Apr;46(4):1707-18.

[20] Liu Y, Yang G, Mirak SA, Hosseiny M, Azadikhah A, Zhong X, Reiter RE, Lee Y, Raman SS, Sung K. Automatic prostate zonal segmentation using fully convolutional network with feature pyramid attention. IEEE Access. 2019 Nov 8;7:163626-32.

[21] Nie D, Shen D. Semantic-guided encoder feature learning for blurry boundary delineation. arXiv preprint arXiv:1906.04306. 2019 Jun 10.

[22] Zhu Q, Du B, Yan P. Boundary-weighted domain adaptive neural network for prostate MR image segmentation. IEEE transactions on medical imaging. 2019 Aug 13;39(3):753-63.

[23] Isensee F, Petersen J, Klein A, Zimmerer D, Jaeger PF, Kohl S, Wasserthal J, Koehler G, Norajitra T, Wirkert S, Maier-Hein KH. nnu-net: Self-adapting framework for u-net-based medical image segmentation. arXiv preprint arXiv:1809.10486. 2018 Sep 27.

[24] Simpson AL, Antonelli M, Bakas S, Bilello M, Farahani K, Van Ginneken B, Kopp-Schneider A, Landman BA, Litjens G, Menze B, Ronneberger O. A large annotated medical image dataset for the development and evaluation of segmentation algorithms. arXiv preprint arXiv:1902.09063. 2019 Feb 25.

[25] Rundo L, Han C, Nagano Y, Zhang J, Hataya R, Militello C, Tangherloni A, Nobile MS, Ferretti C, Besozzi D, Gilardi MC. USE-Net: Incorporating Squeeze-and-Excitation blocks into U-Net for prostate zonal segmentation of multi-institutional MRI datasets. Neurocomputing. 2019 Nov 6;365:31-43.

[26] https://www.overleaf.com/project/607c494fe8d9d7b627cd6869 Zavala-Romero O, Breto AL, Xu IR, Chang YC, Gautney N, Dal Pra A, Abramowitz MC, Pollack A, Stoyanova R. Segmentation of prostate and prostate zones using deep learning. Strahlentherapie und Onkologie. 2020 Oct;196(10):932-42.

[27] Liu Q, Dou Q, Yu L, Heng PA. MS-net: Multi-site network for improving prostate segmentation with heterogeneous MRI data. IEEE transactions on medical imaging. 2020 Feb 17;39(9):2713-24.

[28] Wright, L., 2019. Ranger deep learning optimizer. URL: https://github.com/lessw2020/Ranger-DeepLearning-Optimizer.

[29] Misra D. Mish: A self regularized non-monotonic activation function. arXiv preprint arXiv:1908.08681. 2019 Aug 23.

[30] Gillespie D, Kendrick C, Boon I, Boon C, Rattay T, Yap MH. Deep learning in magnetic resonance prostate segmentation: A review and a new perspective. arXiv preprint arXiv:2011.07795. 2020 Nov 16.

[31] Geert Litjens, Oscar Debats, Jelle Barentsz, Nico Karssemeijer, and Henkjan Huisman. "ProstateX Challenge data", The Cancer Imaging Archive (2017). DOI: 10.7937/K9TCIA.2017.MURS5CL 